solar eclipse visible from the United States. The solar eclipse maps show only the paths of totality, and not the extent of partial phases, but are fascinating nevertheless and are all that is needed for those of us who will travel to view totality. Many of us are already plotting how to view the 23 November 2003 total eclipse that crosses land only over Antarctica.

In The Sun in Eclipse, Michael Maunder and Patrick Moore have written a very practical set of instructions for observing and photographing the eclipse, which fits well in Moore's "Practical Astronomy" series. The information obviously comes from knowledgeable and experienced observers. The discussion of filters and projection methods for solar observation, for example, goes on for 16 pages, and includes careful descriptions of the best filters, a discussion of pinhole projection and a list of "filters which must never be used". Detailed hints on focusing and on the problems of camera shake are wise.

A table lists future eclipses until 2020, but the only map for 11 August is that of totality over Cornwall and Alderney, even though it is so easy to travel from England to the Continent, where weather statistics are more favourable. It is hard to understand why so many people are devoted to seeing the eclipse in England instead of crossing the channel.

Moore has a distillation of his discussion in Patrick Moore's Guide to the 1999 Total Eclipse (Macmillan/Boxtree), whose proceeds go towards building a planetarium in West Sussex. Maps include small versions of the standard ones by Fred Espenak of NASA; they are handy to have, but are not well reproduced, being small and on inexpensive paper.

Sheridan Williams's idiosyncratic UK Solar Eclipses from Year 1 provides maps of all eclipses crossing England over three millennia, and shows how to observe them. Any eclipse buff should have one. Totality: Eclipses of the Sun (2nd edn) by Mark Littman, Fred Willcox and Fred Espenak (Oxford University Press) is late in the publication of eclipse books, but is by very experienced buffs.

The few professional teams of eclipse observers will be overwhelmed by the amateur astronomers and, even more, by the many tourists who will travel into the band of totality. Let us echo Edmond Halley's sentiment from his map predicting the path of the 1715 eclipse over England: "We wish our Astronomical Friends a Clear Sky."

Jay M. Pasachoff is in the Department of Astronomy, Williams College, 3 Lab Campus Drive, Williamstown, Massachusetts 01267, USA, and is Chair of the Working Group on Eclipses of the solar commissions of the International Astronomical Union.

\section{An awkward dinner guest}

\section{Mapping the Mind}

by Rita Carter

Weidenfeld \& Nicolson: 1998. 224 pp. $\mathfrak{E} 25, \$ 29.95$

\section{Jeffrey Gray}

In the early 1970s I had a very bright student, John Churcher, who started doing experimental psychology, then quit to study Chinese, only to come back a term later to psychology. His reason for returning was that the experience of psychology had made it impossible for him to take part in standard dinner-party conversation. His assumptions were just too different from those of everyone else. (In those days there were very few students of psychology or neuroscience.)

I knew just what John meant: it was not so much a question of having different information to bring to bear on the topic of the evening, much more to do with the conceptual framework within which it was approached. Thirty years on, despite the extensive marination of contemporary society in psychology and neuroscience, the 'Churcher gap' is still with us. So, will Rita Carter's beautifully produced and entertainingly written survey of the 'landscape of the brain' help us cross it?

Information there is aplenty, nugget after nugget of it to stimulate and tease the most jaded palate. Many of the nuggets come from recent imaging studies of the human brain in action (prettily illustrated with pictures of brains lighting up here and there in response to this and that). The attentive reader will learn to name particular regions of the brain (amygdala, hypothalamus, hippocampus...) and to attach neat labels to them (fear, sex, memory...). The more attentive reader might, however, wonder whether this kind of paired associate learning throws any real light on the way the brain actually works.

Other nuggets come from the strange vicissitudes that befall people who have had damage to discrete parts of the brain - the kind of case study that Oliver Sacks has made his own - or from various visual and other illusions taken from the treasure-house of psychophysics. Carter does an excellent job of putting together these different kinds of nugget into a unified, diverting and fastmoving narrative.

But, but... the more I read this book, the more concerned I became about the effect it will have on dinner-party conversation. It is, of course, the job of scientific journalism to make science easy to digest, but Carter makes it all too easy. Most of her story is presented as though it were established fact, but very little of it is; and rarely is the reader given any clues for distinguishing well-supported evidence from mere speculation. Above all, one is given the impression that wonderful new machines like PET scanners have opened up startling windows on the brain/mind - lo! We now just have to look in to see what is going on there.

There is hardly any discussion of the way in which behavioural neuroscience sifts evidence, of the conceptual frameworks it uses or the problems they present, or of the difficulties of integrating theory and evidence that come from different starting points. This last issue is fudged to the point of obscurity. There are, for example, long passages that purport to be about brain function, but which are, in fact, filched from common experience and reclothed in spurious neuroscientific talk that only the worker in the field will know has no empirical support.

The conceptual confusions that permeate the book become most worrying when we reach those most likely dinner-party topics: consciousness, free will and the responsibility for one's actions. Neural messages are repeatedly sent from the 'conscious brain' to the 'unconscious brain', or in the other direction, in a way that builds shamelessly upon Cartesian dualist intuitions which are then in the final chapter piously — but without good reason given - dismissed. (The 'conscious brain', by the way, is usually said to lie in the cerebral cortex, sometimes specifically in the frontal cortex; this hypothesis, even if made intelligible, is as yet unsupported by any serious evidence. Equally unsupported is its complement, that everything below the cortex makes up the 'unconscious brain.)

The book relentlessly blurs the critical divide between frank damage to the brain as the clear cause of behavioural derangement and variation in the activity of the brain as a correlate of behavioural variation (a correlation from which, of course, no direction of causality can be inferred). So, towards the end of the book, we are asked to take seriously the hypothesis that the massacres of Armenians by Turks, Jews by Nazis, Cambodians by Pol Pot and Tutsis by Hutus were all brought about by "spasms of overactivity in the orbito-frontal and medial prefrontal cortex".

This absurd extension of neuroscience into history and politics is what I dread most to find at the next dinner party. The Churcher gap, alas, is alive and well.

Jeffrey Gray is at the Institute of Psychiatry and ReNeuron Ltd, De Crespigny Park, Denmark Hill, London SE5 8AF, UK.

Mapping the Mind was short-listed for the 1999 Rhône-Poulenc Prize for Science Books see page 654 . 